\title{
Langmuir-Blodgett films of micron-sized organic and inorganic colloids
}

\author{
Stéphane Reculusa, ${ }^{* a b}$ Romain Perrier-Cornet, ${ }^{b}$ Béatrice Agricole, ${ }^{a}$ \\ Valérie Héroguez, ${ }^{b}$ Thierry Buffeteau ${ }^{c}$ and Serge Ravaine ${ }^{a}$
}

Multilayered films starting with silica or polymer particles in the micron-size range have been prepared using the Langmuir-Blodgett technique. The polymer particles made of highly crosslinked cores and hydrophilic shells were elaborated through a precipitation polymerization method that allows formation of particles with a low polydispersity. The influence of the surface function, the differences between organic and inorganic systems, and the characterization of these materials by means of reflectance infrared spectroscopy are also discussed.

\section{Introduction}

For many years, the Langmuir technique has been thoroughly used to study the behaviour of molecules at several gas-liquid interfaces. ${ }^{1,2}$ However, in the past decades, improvement in nanosciences and experimental techniques leading to nanoparticles of various natures, sizes and functionalities has provided a renewed interest in Langmuir technology, that could bring information on the interfacial behaviour of nanoparticles like clays, ${ }^{3}$ nanowires, ${ }^{4}$ quantum dots, ${ }^{5}$ latexes, ${ }^{6}$ or metal oxides. ${ }^{7}$ For instance, a comparative study could be helpful in predicting the behaviour of particles at liquid-liquid interfaces, such as an oil-water interface, and therefore be useful in the understanding of particle-stabilized systems such as Pickering emulsions. ${ }^{8-10}$

Several techniques have been used so far to get colloidal assemblies such as layer-by-layer, ${ }^{11}$ vertical, ${ }^{12}$ or electrophoretic deposition ${ }^{13}$ but these processes could not be extended to all types of particles. For instance, there is to our knowledge no example of particulate assemblies made of polymer particles with a size bigger than $2 \mu \mathrm{m}$ following one of these strategies. However, for several years, interesting results have been achieved in the elaboration of supraparticulate structures using the Langmuir-Blodgett technique, that could theorically be extended to various systems and particle sizes.

In recent works, several research groups worldwide have demonstrated that the Langmuir-Blodgett (LB) technique was a promising tool in order to elaborate colloidal crystals of silica particles with a well-defined architecture over large dimensions. ${ }^{14-21}$ These previous studies have shown that important criteria are determining the behaviour of these parti-

\footnotetext{
${ }^{a}$ Centre de Recherche Paul Pascal - CNRS, Université Bordeaux 1, Avenue Albert Schweitzer, 33600 Pessac, France. E-mail: reculusa@crpp-bordeaux.cnrs.fr; Fax: + 33 556845600; Tel: +33556845656

${ }^{b}$ Laboratoire de Chimie des Polymères Organiques - CNRS/

Université Bordeaux 1, Ecole Nationale Supérieure de Chimie et de Physique de Bordeaux, 16 avenue Pey Berland, 33600 Pessac, France. Fax: + 33 540008487; Tel: + 33540008486

${ }^{c}$ Institut des Sciences Moléculaires - CNRS/Université Bordeaux 1, 351 cours de la Libération, 33405 Talence Cedex, France
}

cles at the air-water interface and, as a consequence, their ability to form well-organized particulate films at the water surface. First, the diameter $D$ of the particles determines the number of particles that have to be compressed to form a compact Langmuir film with a given area. Indeed, assuming a perfect hexagonal arrangement at the interface, the area per particle is given by the following relationship:

$$
A=\frac{\sqrt{3}}{2} D^{2}
$$

and as a consequence, the amount of particles $m$ required to cover a normalized area of interface $\left(1 \mathrm{~m}^{2}\right)$ is given by:

$$
m=\frac{\pi}{3 \sqrt{3}} \rho D
$$

where $\rho\left(\mathrm{g} \mathrm{cm}^{-3}\right)$ is the volumic mass of the particles. In other words, it is obvious that minimizing the probability of forming defects in particulate Langmuir films is easier with big particles, since less particles, numerically speaking, are required to cover the interface.

\section{Results and discussion}

Due to its easy synthetic routes leading to colloids uniform in shape and size, silica is a naturally good material candidate to study interfacial properties of monodisperse particles. As already observed, the surface chemistry has a strong influence over the quality of a Langmuir film of silica particles. Indeed, the spreading step preliminary to the film formation requires the use of a solvent that is both little-miscible with the subphase (or with a smaller density) and a good dispersant for the particles. For this reason, chloroform-ethanol mixtures are perfect candidates to spread particles at the interface, assuming these particles are properly functionalized to allow dispersion in this solvent mixture. The surface modification versatility of silica, permitted by a sol-gel process implying functional silane derivatives, is consequently very helpful in order to overcome this limitation. ${ }^{22,23}$

As described in our previous works, silica particles with a diameter in the micron-size range can be prepared following a modified Stöber procedure where an alcoholic solution of the silica precursor, tetraethoxysilane, is added in a semi- 


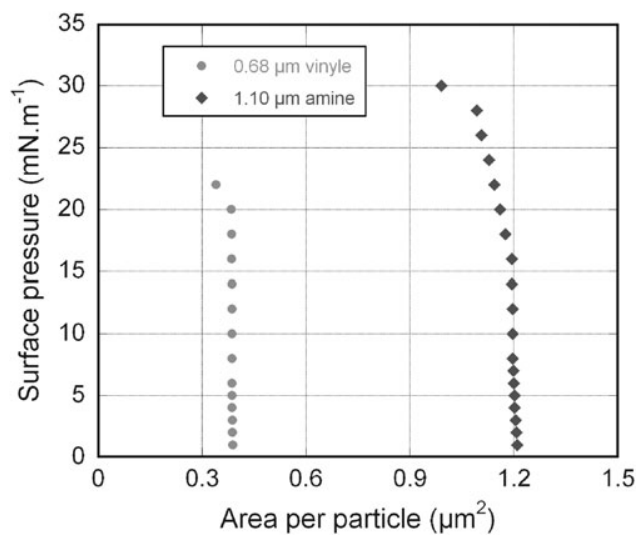

Fig. 1 Compression isotherms of $0.68 \mu \mathrm{m}$ silica particles functionalized using allyltrimethoxysilane (left) and $1.10 \mu \mathrm{m}$ silica particles functionalized using aminopropyl-triethoxysilane (right) at the air-water interface of a Langmuir trough (temperature: $20{ }^{\circ} \mathrm{C}$ ).

continuous fashion into a basic ethanol-water medium. ${ }^{24,25}$ This strategy allows to control quite precisely the final desired size of the silica colloids, ${ }^{26}$ a slow addition rate of the precursor solution also preventing secondary nucleation of particles. Silica particles surface can then be modified using aminopropyltriethoxysilane or allyltrimethoxysilane, thus promoting an appropriate amphiphilic behaviour for the particles' spreading at the water surface. Then, spreading of a given amount of particles in a chloroform-ethanol mixture $(80 / 20$ $\mathrm{v} / \mathrm{v}$ ) was carried out at the water surface of a Langmuir trough under nitrogen atmosphere. As shown in Fig. 1, the compression isotherms of these particles are almost identical to those of smaller particles, with a similar surface hydrophilicity, synthesized in a previous work. ${ }^{27}$ These isotherms are characterized by a very steep slope at low surface pressures, indicating that the particulate films are already well-organized and almost perfectly compact at $1 \mathrm{mN} \mathrm{m}^{-1}$. Increasing the pressure has indeed little effect on the area per particle at the interface, that only starts to decrease above $18 \mathrm{mN} \mathrm{m}^{-1}$, the collapse pressure being reached around $30 \mathrm{mN} \mathrm{m}^{-1}$. According to these results, the pressure chosen for the transfer of the Langmuir film onto hydrophilic substrates was always fixed at $6 \mathrm{mN} \mathrm{m}^{-1}$.

While transferring several layers of particles onto a hydrophilic substrate, it is then possible to elaborate Langmuir-
Blodgett films with a well-defined and homogeneous thickness, ${ }^{14}$ as can be seen on the scanning electron microscope (SEM) pictures presented in Fig. 2. These images were recorded on a JEOL JSM-840 microscope operating at $10 \mathrm{kV}$ after the samples were gold-sputtered.

However, characterization of these Langmuir-Blodgett films is easier with spectroscopic means since we are dealing with materials with a homogeneous thickness. We have already shown that transmission infrared (IR) spectroscopy could be helpful to characterize LB films of silica particles with a size smaller than $1 \mu \mathrm{m}$ but becomes quite limited for bigger particles due to the strong absorbance of silica itself. To circumvent this difficulty, we have taken advantage of the possibility to transfer our LB films onto reflective substrates such as silicon wafers in order to record the reflectance signal of our materials in the IR region.

Reflectance infrared spectra of Langmuir-Blodgett films of silica particles transferred onto silicon wafers are presented in Fig. 3. These spectra were recorded at a spectral resolution of $8 \mathrm{~cm}^{-1}$ using a ThermoElectron Nexus 670 FTIR spectrometer. The reflectance experiments were performed using an external reflection attachment at an angle of incidence of $12^{\circ}$. As shown in Fig. 3a, these spectra are first of all characterized by a main reflectance maximum-between 2 and 2.5 $\mu \mathrm{m}$-whose position and half-height width are better and better defined when the number of layers increases. This maximum corresponds to the Bragg peak whose wavelength position depends on both the particle size and the mean refractive index of the medium, $n_{\mathrm{eff}}$ (close to 1.33 for a periodic arrangement of silica spheres in the air). Indeed, according to Bragg's law:

$$
\lambda_{\mathrm{B}}=2 n_{\mathrm{eff}} \sqrt{\frac{2}{3}} D
$$

From the best resolved Bragg peaks in graph 3a (corresponding to the thickest samples), we can estimate that $\lambda_{\mathrm{B}}=$ $2.27 \mu \mathrm{m}$, what leads to a calculated $D$ value around $1.05 \mu \mathrm{m}$, very close to the diameter estimated by SEM.

As described in some of our previous works, IR spectra of such LB films can also present some interesting features, as shown in Fig. 3b. In the wavelength region between 3 and $8 \mu \mathrm{m}$, remarkable periodic Fabry-Pérot fringes appear within the different spectra, thus modifying the IR spectrum of silica
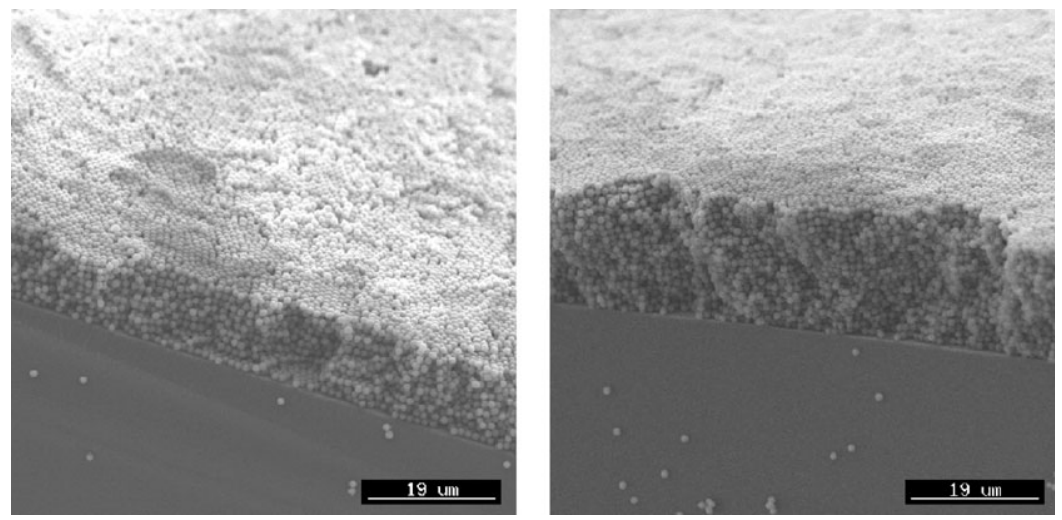

Fig. 2 SEM pictures of LB films of $1.10 \mu \mathrm{m}$ silica particles deposited onto glass substrates with 10 (left) and 20 layers (right). 

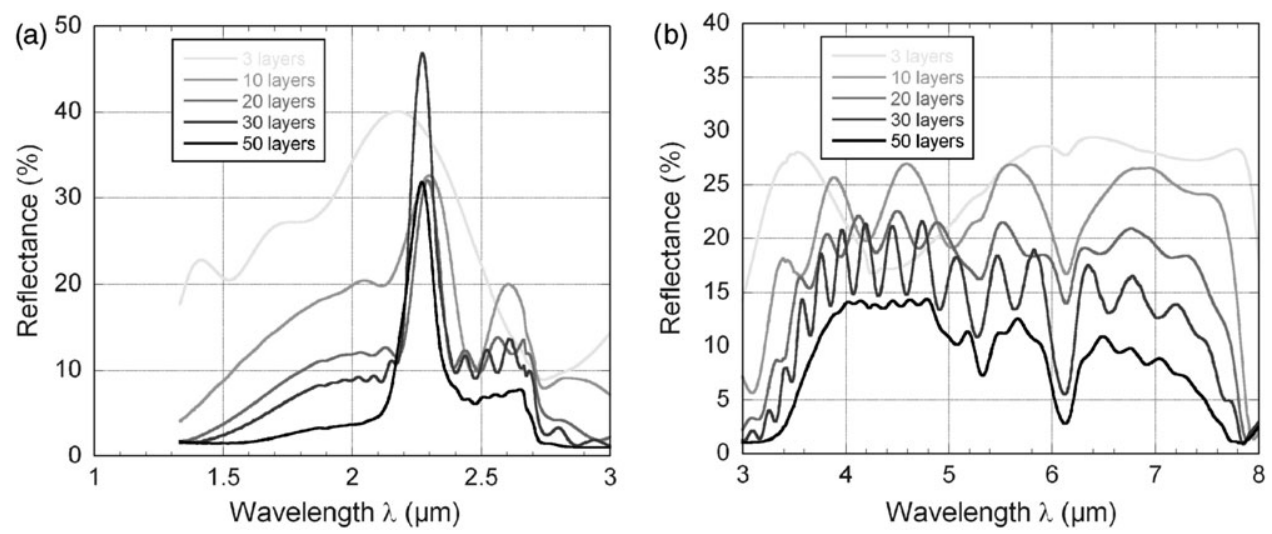

Fig. 3 Reflectance IR spectra of LB films of various thicknesses onto silicon wafers: (a) IR region of the Bragg peak position; (b) IR region where Fabry-Pérot fringes appear.

itself. When the LB film thickness increases, the amplitude and the period of the fringes decreases in a regular fashion, what indicates the homogeneity of the particulate deposit thickness, i.e. its regular layer-by-layer arrangement. In Fig. 4, we have represented some experimental data depending on the positions of these fringes (for a given thickness, $\lambda_{0}$ is the wavelength of the first fringe and the $\lambda_{\mathrm{m}}$ are the positions of the following ones). For all the samples, the linear fit is almost perfect with regression coefficients higher than 0.999 whereas the slopes simply correspond to the LB film thickness. As shown in Table 1, the experimental values are very close to the theoretical ones, calculated using the following relationship: ${ }^{14}$

$$
\theta(N)=\left[1+(N-1) \times \sqrt{\frac{2}{3}}\right] \times D
$$

where $\theta$ is the film thickness and $N$ the number of deposited layers. Moreover, the deviation of the experimental value in respect to the theoretical one decreases rapidly when the number of layers increases, due to the higher accuracy over the fringes positions.

Despite the simplicity of the involved chemical reactions, obtaining monodisperse silica particles with a size bigger than $1 \mu \mathrm{m}$ through a sol-gel route is a long and time-consuming process. In order to study the interfacial behaviour of larger particles, that could also present the considerable advantage of

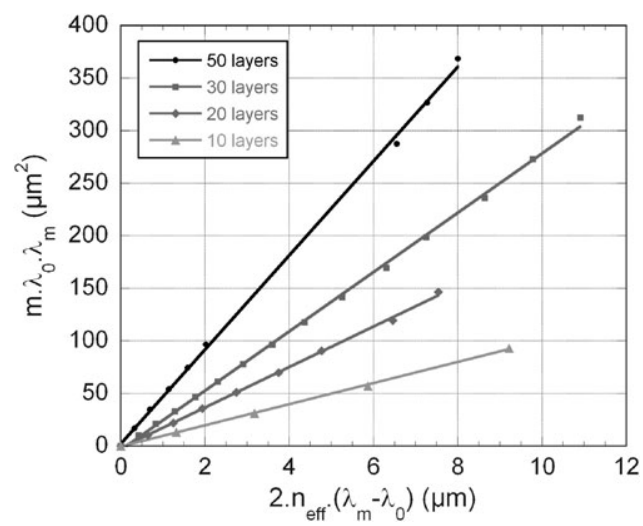

Fig. 4 Calculation of Langmuir-Blodgett film thicknesses starting from the Fabry-Pérot fringes positions. being easily observed by classical optical microscopy, we were interested by synthetic procedures leading to micronic and monodisperse polymer particles, whose surface properties could be controlled through a well-chosen mixture of monomers. The polymer particles used in this work were prepared through a two-step polymerization process. First, cross-linked core particles were prepared through precipitation polymerization of a mixture of monomers, divinylbenzene (DVB) and ethyleneglycol dimethacrylate (EGDMA), in acetonitrile, following a procedure described elsewhere. ${ }^{28}$ These highly crosslinked cores ensure a good chemical resistance in all of the most common solvents, and chloroform in particular, what is a necessary prerequisite of the spreading process in the Langmuir film preparation. A co-monomer such as chloromethylstyrene could also be included in the mixture of monomers, which provides some chlorine functions at the core particles surface. ${ }^{29}$ These reactive groups are then useful in the second step consisting of an atom transfer radical polymerization of tert-butylacrylate in presence of a $\mathrm{CuBr}$ /pentamethyl-diethylenetriamine mixture catalyst, leading to the formation af a hairy polymer shell. Following deprotection of carboxylic acid functions through acidolysis of the tert-butyl groups in dichloromethane in presence of trifluoroacetic acid finally leads to polymer particles with an amphiphilic macroscopic behaviour, due to the hydrophobic core/hydrophilic shell structure combination. ${ }^{30}$ This property is illustrated on the macroscopic scale by the easy dispersion of the particles in water, ethanol or chloroform.

Polymer bead suspensions with a concentration around $20 \mathrm{mg} \mathrm{mL}{ }^{-1}$ were prepared by weighing a given amount of particles and dispersing them in a mixture of ethanol and chloroform $(80 / 20 \mathrm{v} / \mathrm{v})$. Spreading and compression at the air-water interface were carried out following an experimental procedure identical to the one previously described with silica particles.

Table 1 Values of thickness of several LB films of silica particles

\begin{tabular}{lccc}
\hline & \multicolumn{2}{c}{ Sample thickness/ $\mu \mathrm{m}$} & \\
\cline { 2 - 3 } Number of layers & Theoretical & Experimental & Deviation \\
\hline 10 & 9.18 & 10.04 & $+9.37 \%$ \\
20 & 18.16 & 19.26 & $+6.06 \%$ \\
30 & 27.15 & 28.17 & $+3.76 \%$ \\
50 & 45.11 & 44.80 & $-0.69 \%$ \\
\hline
\end{tabular}




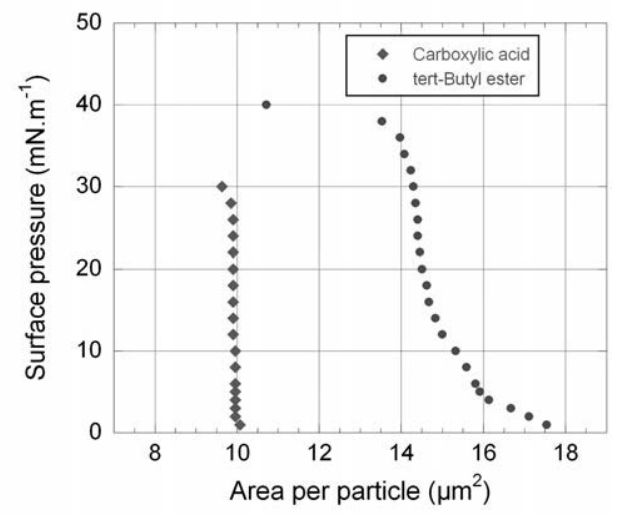

Fig. 5 Compression isotherms of Langmuir films of polymer particles with different surface functions (with or without deprotection of the carboxylic acid groups).

Compression isotherms pictured in Fig. 5 were recorded through a manual and stepwise compression of the Langmuir films of particles at $20{ }^{\circ} \mathrm{C}$ and under a nitrogen atmosphere. The amount of spread particles was identical in both cases ( $c a$. $120 \mathrm{mg}$ ) and the area per particle was calculated assuming a particle diameter around $3.8 \mu \mathrm{m}$ and a mean volumic mass around $1.19 \mathrm{~g} \mathrm{~cm}^{-3}$. The isotherm of the surface hydrophilic particles $(\bullet)$ is very similar to the isotherm already observed with silica particles leading to a homogeneous and wellorganized monolayer of particles with a very steep slope at low surface pressures and a collapse pressure around $30 \mathrm{mN} \mathrm{m}^{-1}$. On the contrary, the behaviour of non-deprotected particles, i.e. with a hydrophobic shell $(-)$, is completely different since the area per particle varies significantly until a pressure of around 20 $\mathrm{mN} \mathrm{m}^{-1}$. Moreover, whereas the size and the amount of particles were the same in both cases, one can notice that the surface area per particle is more or less 1.5 times higher in the case of hydrophobic particles, which should indicate that the particulate film is much less dense compared to the hydrophilic one.

As pictured in Fig. 6, observation of monolayers of polymer particles transferred onto hydrophilic glass slides illustrates perfectly the influence of the surface function of a particle on the two-dimensional organization at the air-water interface. Indeed, at the same transfer pressure of $6 \mathrm{mN} \mathrm{m}^{-1}$ polymer particles with tert-butyl ester groups at their surface-i.e. undeprotected hydrophobic functions-form a "patchy" film. In this 2D structure, some well-packed and organized-but small - domains coexist with individual particles as well as non close-packed areas. On the opposite, polymer particles whose surface functions have been deprotected through acidolysis, consequently exhibiting hydrophilic carboxylic acid groups at their surface, provide a much more dense particulate film. This result first confirms the macroscopic observation that deprotected particles are easily dispersible in water, unlike their undeprotected analogues. Secondly, it provides a clear demonstration that the surface function is the main criterium that determines the formation of a well-organized array of particles, whereas the density or the hydrophobicity of the core has little influence.
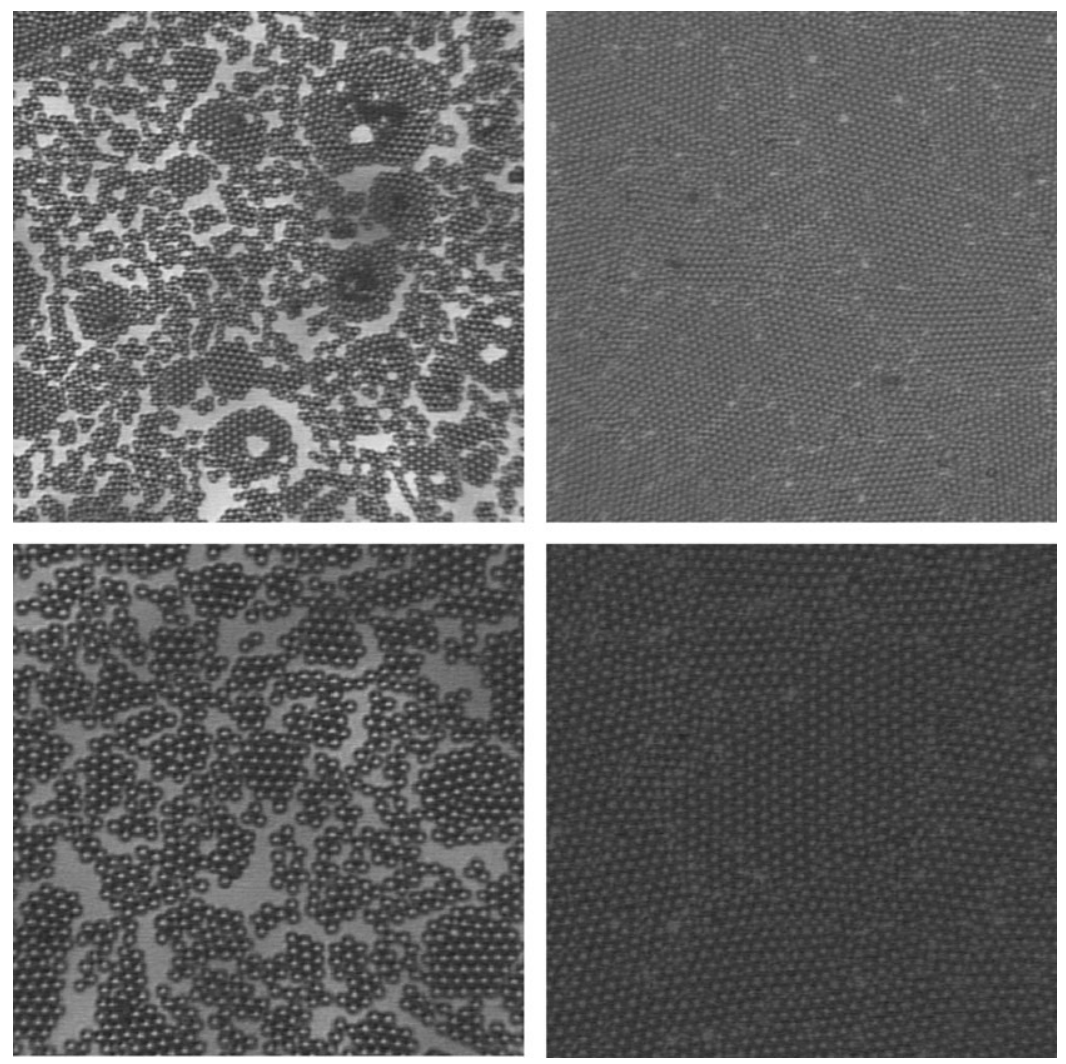

Fig. 6 Optical microscope views of monolayers of poly(DVB-EGDMA) particles transferred onto glass substrates at $6 \mathrm{mN} \mathrm{m}^{-1}$. Left: particles with hydrophobic tert-butyl ester groups at their surface; right: particles with hydrophilic carboxylic acid groups after deprotection through acidolysis. Particle diameter: $3.8 \mu \mathrm{m}$. 

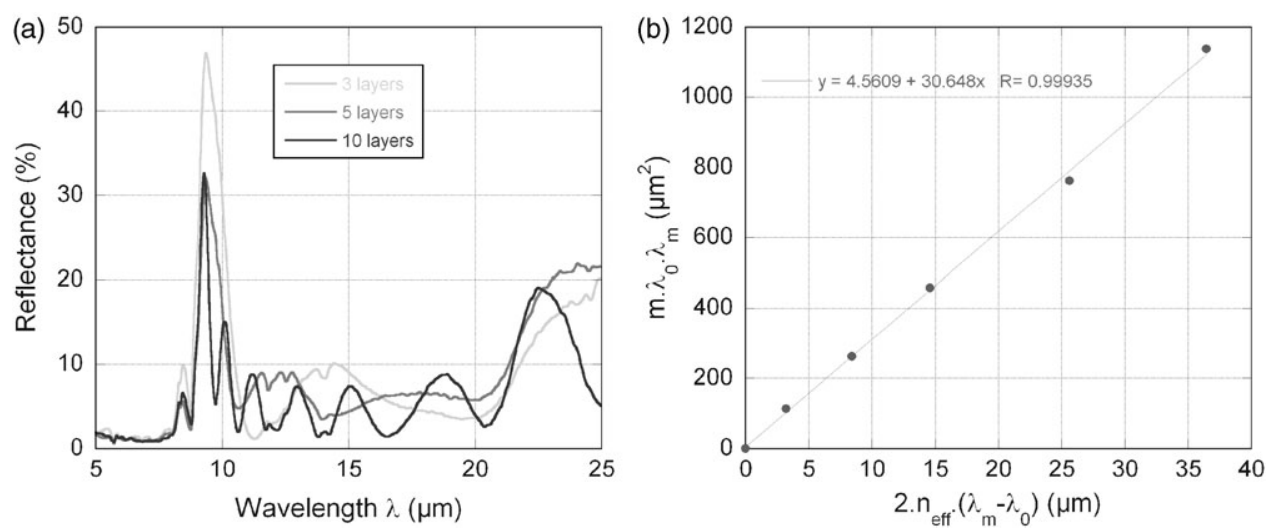

Fig. 7 (a) Reflectance spectra of LB films of surface-hydrophilic poly(DVB-EGDMA) particles of various thicknesses onto glass substrates; (b) Thickness calculation from the Fabry-Pérot fringes positions for the 10-layers sample.
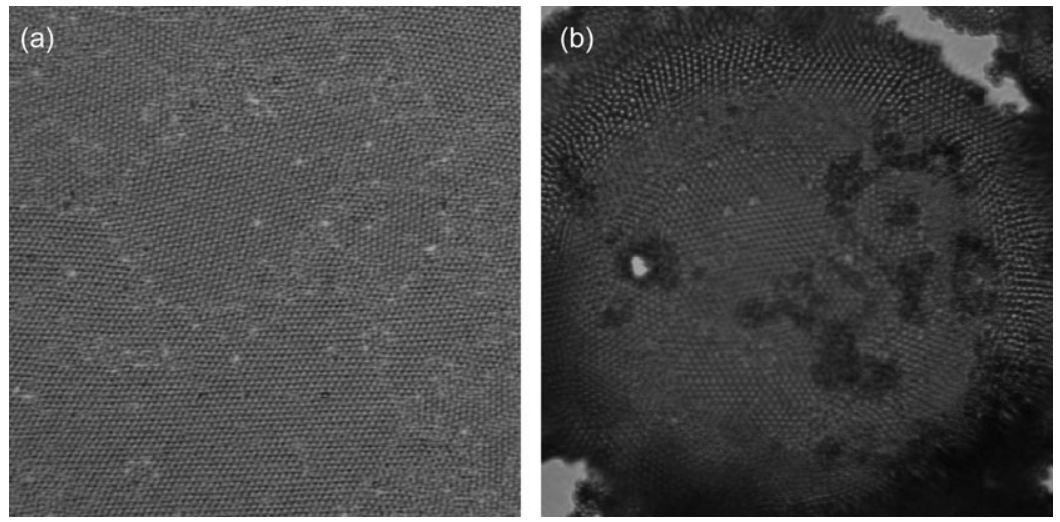

Fig. 8 Optical microscopy images of a single layer of polymeric particles with a highly cross-linked poly(DVB-EGDMA) core and a hydrophilic shell: monolayer transferred onto a glass substrate through the LB technique (left); surface-stabilized oil-in-water droplet (right). Particle diameter: $3.5 \mu \mathrm{m}$.

Langmuir-Blodgett films consisting of surface hydrophilic polymer particles were also successfully obtained by sequential deposition of the particulate monolayer onto hydrophilic glass slides. Due to the big particle diameter, it is however not possible to observe the Bragg diffraction peak by conventional IR transmission experiments, as we did previously with submicronic silica particles. Indeed, the effective refractive index $n_{\text {eff }}$ for a f.c.c. arrangment of polymer particles $(n \sim 1.6)$ in the air $(n \sim 1)$ should be around 1.47. As a consequence, following eqn (2), the theoretical Bragg peak position should be close to $1100 \mathrm{~cm}^{-1}$ (i.e. $\lambda_{\mathrm{B}}=9.1 \mu \mathrm{m}$ ), in a wavelength region where glass absorbs strongly the IR radiation. Fortunately, reflectance IR spectroscopy is again helpful in this precise case since the strong absorption of IR radiation in this spectral domain ensures a good reflectance signal of our polymer films deposited onto glass slides. ${ }^{31}$

As represented in Fig. 7a for three different samples, the recorded reflectance IR spectra still allow to estimate both the particles diameter starting from the Bragg peak position $\left(\lambda_{\mathrm{B}} \sim\right.$ $9.29 \mu \mathrm{m})$ as well as the LB film thickness. For the thickest sample, the Fabry-Pérot fringes are also well-defined and the linear fit provided in Fig. $7 \mathrm{~b}$ is almost perfect. From the calculated slope of $30.65 \mu \mathrm{m}$, one could then deduce, using eqn (3), a diameter $D$ of around $3.67 \mu \mathrm{m}$, which is quite close to the awaited value of $3.8 \mu \mathrm{m}$, taking into account the uncertainties on the exact fringes positions as well as the real refractive index of our material.

From these results, one can infer that the elaboration of a multilayered structure of micron-sized polymer particles through the LB technique has been successful. As said previously, this achievement was made possible by the appropriate amphiphilic behaviour of these particles and their ability to form a well-organized Langmuir film after compression. As shown in Fig. 8, particles forming well-ordered arrays at the air-water interface seem to have similar properties at the curved interfaces of oil-in-water droplets, as described more thoroughly by Leal Calderon and co-workers in another paper of this special PCCP issue. ${ }^{32}$ Excepted from the Pickering emulsions area, such particles could also be particularly useful in order to elaborate 3-dimensional ordered arrays of particles whose porous replicas, that would present micron-sized voids after the template removal ${ }^{33}$ could be of interest for catalytic purposes ${ }^{34}$ or cellular scaffolds, ${ }^{35}$ which cannot be obtained using smaller particles as basic units.

\section{Conclusions}

In this paper, we have reminded some of the important parameters involved in the formation of Langmuir films of particles and detailed more precisely the case of micron-sized 
particles. We have demonstrated that the Langmuir-Blodgett technique might be of high interest in order to elaborate $3 \mathrm{D}$ assemblies of micron-sized particles, since reflectance IR spectroscopy results indicate that materials with a thickness up to $50 \mu \mathrm{m}$ could be obtained through this strategy. Results obtained with large polymer particles with an appropriate core-shell structure indicate the extensibility of this strategy to chemical systems different from silica and, most interestingly, to particles with a size much bigger than those used in past works.

\section{Acknowledgements}

The authors would like to thank Elisabeth Sellier (Centre de Ressources en Microscopie Electronique et Microanalyse, Université Bordeaux 1, Talence) for her help in SEM experiments.

\section{References}

1 K. B. Blodgett, J. Am. Chem. Soc., 1935, 57, 1007.

2 G. G. Roberts, Adv. Phys., 1985, 34, 475.

3 R. H. A. Ras, Y. Umemura, C. T. Johnston, A. Yamagishi and R. A. Schoonheydt, Phys. Chem. Chem. Phys., 2007, 9, 918.

4 A. Tao, F. Kim, C. Hess, J. Goldberger, R. He, Y. Sun, Y. Xia and P. Yang, Nano Lett., 2003, 3, 1229.

5 Y.-J. Shen, Y.-L. Lee and Y.-M. Yang, J. Phys. Chem. B, 2006, 110, 9556.

6 K.-U. Fulda and B. Tieke, Supramol. Sci., 1997, 4, 265.

7 L. Naszályi, A. Deák, E. Hild, A. Ayral, A. L. Kovács and Z. Hórvölgyi, Thin Solid Films, 2006, 515, 2587.

8 S. U. Pickering, J. Chem. Soc., Trans., 1907, 91, 2001.

9 N. P. Ashby and B. P. Binks, Phys. Chem. Chem. Phys., 2000, 2, 5640.

10 B. P. Binks and S. O. Lumsdon, Langmuir, 2001, 17, 4540.

11 K. P. Velikov, C. G. Christova, R. P. A. Dullens and A. Van Blaaderen, Science, 2002, 296, 106.

12 P. Jiang, J. F. Bertone, K. S. Hwang and V. L. Colvin, Chem. Mater., 1999, 11, 2132.
13 A. L. Rogach, N. A. Kotov, D. S. Koktysh, J. W. Ostrander and G. A. Ragoisha, Chem. Mater., 2000, 12, 2721.

14 S. Reculusa and S. Ravaine, Chem. Mater., 2003, 15, 598.

15 P. Massé, S. Reculusa, K. Clays and S. Ravaine, Chem. Phys. Lett., 2006, 422, 251.

16 M. E. Pemble, M. Bardosova, I. M. Povey, R. H. Tredgold and D. Whitehead, Physica B, 2007, 394, 233.

17 M. Bardosova, P. Hodge, V. Smatko, R. H. Tredgold and D. Whitehead, Acta Phys. Slov., 2004, 54, 409.

18 M. Szekeres, O. Kamalin, P. G. Grobet, R. A. Schoonheydt, K. Wostyn, K. Clays, A. Persoons and I. Dekany, Colloids Surf., A, 2003, 227, 77 .

19 A. Deák, B. Bancsi, A. L. Tóth, A. L. Kovács and Z. Hórvölgyi, Colloids Surf., A, 2006, 278, 10.

20 A. Gil and F. Guitian, J. Colloid Interface Sci., 2007, 307, 304.

21 Y.-L. Lee, Z.-C. Du, W.-X. Lin and Y.-M. Yang, J. Colloid Interface Sci., 2006, 296, 233.

22 F. Caruso, Adv. Mater., 2001, 13, 11.

23 W. Wang and B. Gu, J. Phys. Chem. B, 2005, 109, 22175.

24 W. Stöber, A. Fink and E. Bohn, J. Colloid Interface Sci., 1968, 26, 62 .

25 S. Kang, S. I. Hong, C. R. Choe, M. Park, S. Rim and J. Kim, Polymer, 2001, 42, 879.

26 S. Reculusa, C. Poncet-Legrand, S. Ravaine, C. Mingotaud, E. Duguet and E. Bourgeat-Lami, Chem. Mater., 2002, 14, 2354.

27 S. Reculusa and S. Ravaine, Appl. Surf. Sci., 2005, 246, 409.

28 W.-H. Li, K. Li and H. D. H. Stöver, J. Polym. Sci., Part A, 1999, 37, 2295.

29 J. S. Downey, R. S. Frank, W.-H. Li and H. D. H. Stöver, Macromolecules, 1999, 32, 2838.

30 R. Perrier-Cornet and V. Héroguez, unpublished results.

31 T. Buffeteau and B. Desbat, Appl. Spectrosc., 1989, 43, 1027.

32 F. Gautier, M. Destribats, R. Perrier-Cornet, J.-F. Dechezelles, J. Giermanska, V. Héroguez, S. Ravaine, F. Leal-Calderon and V. Schmitt, Phys. Chem. Chem. Phys., in this issue.

33 M. L. K. Hoa, M. Lu and Y. Zhang, Adv. Colloids Interface Sci., 2006, 121, 9.

34 R. Szamocki, S. Reculusa, S. Ravaine, P. N. Bartlett, A. Kuhn and R. Hempelmann, Angew. Chem., Int. Ed., 2006, 45, 1317.

35 Y. Liu, S. Wang, J. W. Lee and N. A. Kotov, Chem. Mater., 2005, 17, 4918 . 